\title{
Antiferromagnetic coupling of Cr-porphyrin to a bare Co substrate
}

\author{
Jan Girovsky, ${ }^{1, *}$ Kartick Tarafder, ${ }^{2,3}$ Christian Wäckerlin, ${ }^{1}$ Jan Nowakowski, ${ }^{1}$ Dorota Siewert, ${ }^{4}$ Tatjana Hählen, ${ }^{1}$ \\ Aneliia Wäckerlin, ${ }^{4}$ Armin Kleibert, ${ }^{5}$ Nirmalya Ballav, ${ }^{6}$ Thomas A. Jung, ${ }^{1}$ and Peter M. Oppeneer ${ }^{2, \dagger}$ \\ ${ }^{1}$ Laboratory for Micro- and Nanotechnology, Paul Scherrer Institute, CH-5232 Villigen PSI, Switzerland \\ ${ }^{2}$ Department of Physics and Astronomy, Uppsala University, Box 516, S-751 20 Uppsala, Sweden \\ ${ }^{3}$ Department of Physics, BITS-Pilani Hyderabad Campus, Shameerpet, Andhra Pradesh 500078, India \\ ${ }^{4}$ Department of Physics, University of Basel, CH-4056 Basel, Switzerland \\ ${ }^{5}$ Swiss Light Source, Paul Scherrer Institute, CH-5232 Villigen PSI, Switzerland \\ ${ }^{6}$ Department of Chemistry, Indian Institute of Science Education and Research, Pune 411008, India \\ (Received 19 March 2014; revised manuscript received 10 November 2014; published 10 December 2014)
}

\begin{abstract}
We report the discovery of an antiferromagnetic coupling of the magnetic moment of chromium(II) tetraphenylporphyrin (CrTPP) molecules to the magnetization of the clean ferromagnetic $\mathrm{Co}(001)$ substrate. We assign this unusual molecule-substrate exchange coupling to the less than half-filled chromium $3 d$ orbitals interacting with Co valence band electrons via porphyrin-ligand molecular orbitals. X-ray magnetic circular dichroism, $\mathrm{x}$-ray photoelectron spectroscopy, and scanning tunneling microscopy are combined with DFT $+U$ calculations and provide evidence for a surprising type of antiferromagnetic $90^{\circ}$ indirect magnetic exchange coupling.
\end{abstract}

Spin-bearing metal-organic molecules have attracted considerable attention in recent years [1-4]. In particular, squareplanar coordination complexes offer a wide range of tunable chemical functionalities [5-8], which derive from the sensitive interaction of the open shell of the metal center with the planar ligands as well as with the surrounding molecular architecture. In "on-surface" configurations novel properties can arise when the magnetic and electronic characteristics of the metal-organic species are subtly modified by the surface-molecule interaction. Accordingly, it was discovered that metallophthalocyanines and metalloporphyrins with $3 d$ and $4 f$ block metal centers exhibit remarkable electronic, optoelectronic, and magnetic properties when adsorbed on surfaces [1,2,5-16]. Strikingly, the magnetic exchange coupling of the spin of metalloporphyrins or metallophthalocyanines with that of ferromagnetic (FM) substrates (Co and $\mathrm{Ni}$ ) was found to induce stable magnetic order in the paramagnetic molecules at and above room temperature $[1,2,11]$. It was reported that metallophthalocyanines and metalloporphyrins align their magnetic moments parallel to those of the bare FM substrate. The origin of this alignment has been much discussed $[1,2,7,10,11]$ and assigned either to direct or indirect magnetic exchange coupling via the chemical bonds across the interface. Understanding and controlling molecule-substrate interactions are key ingredients for designing novel functional surfaces [15].

To date, an antiferromagnetic (AFM) indirect exchange interaction has been reported for planar molecules assembled on oxygen reconstructed $\mathrm{Ni}$ and Co substrates $[5,6]$ or when a graphene interlayer [12] was introduced between the molecule and substrate. An indirect AFM coupling was also observed for the rare-earth double-decker Tb-bis-phthalocyanine adsorbed on a metallic FM substrate, where one macrocycle acts as an interlayer [13]. However, for transition-metal porphyrins and phthalocyanines on bare FM substrates an AFM coupling has not yet been observed.

\footnotetext{
*jan.girovsky@psi.ch

†peter.oppeneer@physics.uu.se
}

Here we report an AFM coupling of $\mathrm{Cr}(\mathrm{II})$ tetraphenylporphyrin molecules adsorbed on bare FM Co. We describe this discovery by a $90^{\circ}$ indirect exchange coupling between the less than half-filled $3 d$ shell of the $\mathrm{Cr}$ ion and $\mathrm{Co}$ substrate mediated by nitrogen atoms in the porphyrin ligand. Such an AFM interaction of square-planar metal-organic complexes provides a showcase for the exchange coupling at substrate-organic spin interfaces and offers opportunities for tuning this interaction, e.g., for spintronics applications.

The sample preparation is carried out in situ under ultrahigh vacuum conditions with a base pressure $p \leqslant 5 \times$ $10^{-10}$ mbar. The FM fcc Co(001) thin films [ 20 monolayers (ML)] are grown by $e$-beam evaporation onto clean $\mathrm{Cu}(001)$ surfaces [6]. The surface chemical composition is monitored at each preparation step by x-ray photoelectron spectroscopy (XPS). The thickness of the Co film and of the molecular layer is controlled by a quartz crystal microbalance and by XPS. $\mathrm{Cr}(\mathrm{III})$ tetraphenylporphyrin chloride $(\mathrm{CrTPPCl})$ molecules are thermally sublimed at a temperature of $\sim 500 \mathrm{~K}$ onto the $\mathrm{Co}(001)$ substrate, which is kept at room temperature. The molecules transform upon contact with the substrate into CrTPP by release of the $\mathrm{Cl}$ ligand [see below-cf. the inset of Fig. 1(a)].

The electronic and magnetic properties of CrTPP molecules on a Co substrate are investigated by element-specific $\mathrm{x}$-ray absorption (XA) and $\mathrm{x}$-ray magnetic circular dichroism (XMCD) spectroscopy in total electron yield mode [Fig. 1(a)]. The chosen experimental geometry with a $\theta_{k}=70^{\circ}$ angle between the x-ray's propagation vector $\vec{k}$ and the surface normal $\vec{n}$ predominantly probes the in-plane components of the magnetic moments of the substrate (white arrows) and of the molecules (yellow arrows). The magnetization of the substrate is reversed by applying an external magnetic field $(|\vec{B}| \sim 120 \mathrm{mT})$. Due to the shape anisotropy the Co film saturates the magnetization along its [110] direction [16]. The $\mathrm{XA} / \mathrm{XMCD}$ spectra are recorded in remanence at the Co and Cr $L_{3,2}$ edges using circularly polarized x-rays of opposite helicities, denoted as $\mathrm{C}^{+}$and $\mathrm{C}^{-}$(see Fig. 1). The Co XMCD signal [see Fig. 1(b)], given by the difference of $\mathrm{C}^{+}$and $\mathrm{C}^{-}$, 


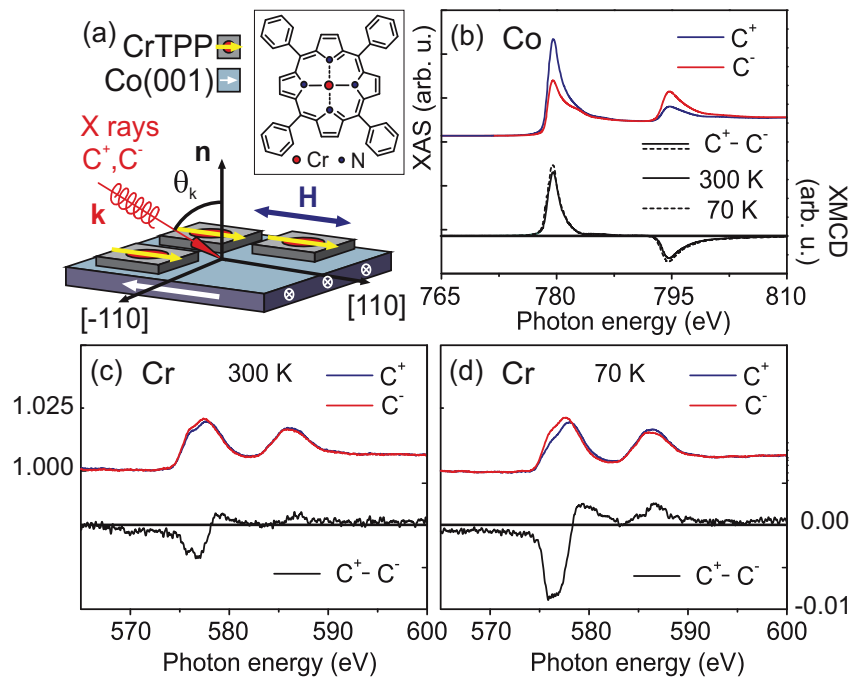

FIG. 1. (Color online) (a) Sketch of the experimental setup with the CrTPP molecules lying flat on the $\mathrm{Co}(001)$ substrate. Prior to the measurements the sample is magnetized by an external magnetic field along the $\mathrm{Co}[110]$ direction. The [110] direction of the substrate, surface normal $\vec{n}$, and propagation vector $\vec{k}$ of the circularly polarized $\mathrm{x}$ rays lies in one plane. The AFM coupling of the molecules with respect to the substrate is depicted by the yellow and white arrows. A scheme of CrTPP is shown in the inset. (b) XA (red and blue lines) and XMCD spectra (black and dashed lines) acquired at the Co $L_{3,2}$ edges of the substrate, at 300 and $70 \mathrm{~K}$. (c), (d) XA and XMCD spectra of the CrTPP molecules measured at the $\mathrm{Cr} L_{3,2}$ edges at 300 and $70 \mathrm{~K}$, respectively.

confirms the magnetic saturation of the Co film. Cooling from $300 \mathrm{~K}$ down to $70 \mathrm{~K}$ reveals a slightly increased XMCD magnitude which reflects a temperature-dependent saturation of the Co magnetization [5]. In the same configuration at 300 $\mathrm{K}$ the relatively weak XMCD signal at the $\mathrm{Cr} L_{3,2}$ edge reveals an unexpected antiparallel orientation of the CrTPP magnetic moment with respect to the substrate magnetization [Fig. 1(c)]. The magnitude of the Cr XMCD signal increases by a factor of 2 at $70 \mathrm{~K}$ [Fig. 1(d)] when compared to $300 \mathrm{~K}$, suggesting a relatively weak exchange coupling.

Analyzing the XA spectra at the $\mathrm{Cr} L_{3,2}$ edges reveals broad features with two well resolved peaks at photon energies of 576.0 and $577.5 \mathrm{eV}$, respectively. The position and shape of the XA peaks support a $2+$ oxidation state of the $\mathrm{Cr}$ ion (cf. Ref. [17]). This is in agreement with Refs. [16,18], where it was found that similar molecules, manganese(III) tetraphenylporphyrin chloride (MnTPPCl), lose their chlorine ligand upon adsorption onto a bare Co substrate and thereby change their oxidation state from $3+$ to $2+$.

The chemical state of the $\operatorname{CrTPP}(\mathrm{Cl})$ molecules and its modification is further probed by XPS [Figs. 2(a) and 2(a)] using monochromatized $\mathrm{Al} K \alpha$ x rays. The multilayer $(\sim 10 \mathrm{ML})$ of $\mathrm{CrTPPCl}$ molecules on $\mathrm{Au}(111)$ shows the $\mathrm{Cr} 2 p_{3 / 2}$ core level peak at a binding energy of $E_{B} \sim 576.8 \mathrm{eV}$ [Fig. 2(a), blue dots], indicating a $3+$ oxidation state of $\mathrm{Cr}$ due to the presence of the axial chloride ligand. In contrast, the $\mathrm{Cr} 2 p_{3 / 2}$ peak for the single CrTPP layer on $\operatorname{Co}(001)$ is found at a lower binding energy $E_{B} \sim 575.0 \mathrm{eV}$, which reveals a change of the $\mathrm{Cr}$ oxidation state from $3+$ in the multilayer to $2+$ in the
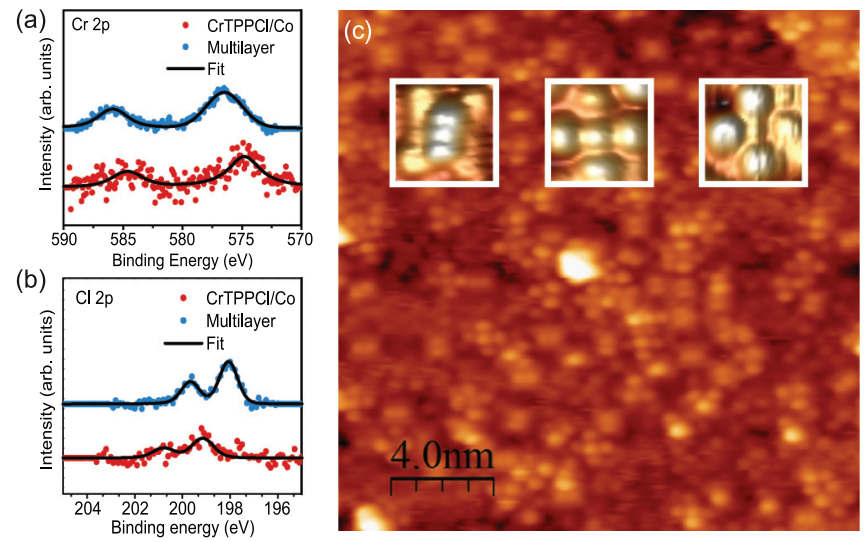

FIG. 2. (Color online) XPS data measured at the $2 p_{3 / 2}$ core levels of (a) $\mathrm{Cr}$ and (b) $\mathrm{Cl}$ for a single layer of CrTPP molecules on $\mathrm{Co}(001)$ (red dots) and a multilayer of CrTPPCl on Au(111) (blue dots). The solid lines give the results of a Gaussian-Lorentzian fit of the spectra. (c) STM data of CrTPP molecules on $\operatorname{Co}(001)$. Three different characteristic patterns are presented in the insets and are assigned to different conformations of the molecules (6-lobe, 5-lobe, 4-lobe, from left to right). ( $U_{\text {bias }}=-2.1 \mathrm{~V}, I_{t}=-10 \mathrm{pA}, T=300 \mathrm{~K}$.)

monolayer on a Co substrate. Note that for $\mathrm{Cl}$ present between the $\mathrm{Cr}$ and $\mathrm{Co}$ atoms, the observed $\mathrm{Cr}$ oxidation state is $3+$ (see Fig. S1 of the Supplemental Material [19]). The $\mathrm{Cl} 2 p_{3 / 2}$ peak position in our XPS data of the CrTPPCl multilayer on $\mathrm{Au}(111)$ is found at a lower energy compared to the CrTPP single layer on $\operatorname{Co}(001)$ [Fig. 2(b)]. This chemical shift confirms that chlorine is detached from CrTPPCl upon adsorption and directly adsorbed on the Co substrate [16]. As also evidenced by our data, $\mathrm{Cl}$ does not contribute to the AFM exchange coupling between CrTPP molecules and the bare cobalt.

Scanning tunneling microscopy (STM) provides information (cf. Ref. [20]) on the orientation and shape of the CrTPP molecules adsorbed on a $\mathrm{Co}(001)$ thin film [Fig. 2(c)]. STM resolves three different conformations characterized by six, five, and four lobes, respectively [Fig. 2(c), left to right inset]. The bright spots at the sides of the four- and five-lobed species are identified as the phenyl rings of the TPP ligand. The six-lobed conformation with a rectangular shape is attributed to a saddle-shape conformation [Fig. 2(c), left inset] which is characterized by a tetrahedral distortion of the porphyrin macrocycle upon adsorption, in analogy to Co(II)TPP on $\operatorname{Ag}(111)$ [21]. A statistical analysis performed on large-scale STM micrographs (see Fig. S2 of the Supplemental Material [19]) revealed that the four-lobed species [Fig. 2(c), right inset] appears in less than $\sim 10 \%$ of the cases. This minority conformer is attributed to axially ligated molecules in analogy to Refs. [16,18]. The conformers imaged as five- or as six-lobed species [Fig. 2(c), center and left inset] are equally frequent ( $\sim 45 \%$ vs $\sim 45 \%$ ). The observation of only a $2+$ oxidation state in Cr XPS and XAS (see above) in the case of a single adlayer provides the evidence that both these conformers, but not necessarily the four-lobed minority conformer, contain a $\mathrm{Cr}$ (II) ion in the center of the porphyrin [Fig. 2(a)]. This assignment is consistent with an earlier report on similar MnTPP molecules on Co, where five- and four-lobed species have been attributed to the molecules containing $\mathrm{Mn}$ (II) and 
Mn(III) ions, respectively [16]. According to our STM data, the different conformers adsorb in different orientations: The five-lobed species of CrTPP adsorbs on the $\mathrm{Co}(001)$ surface with the N-Cr-N parallel to the substrate's [010] and [100] crystallographic axes (see Fig. S3 of the Supplemental Material [19]). The rectangular shape of the six-lobed species preordains these molecules to adsorb in two different orientations which are equivalent with respect to the substrate symmetry. The six-lobed molecules align their $\mathrm{N}-\mathrm{Cr}-\mathrm{N}$ coordination bond direction parallel to the easy axes of the magnetization of the substrate. Density functional theory $(\mathrm{DFT}+U)$ calculations reveal that for the six-lobed species the porphyrin nitrogens sit on top of Co substrate atoms while the $\mathrm{Cr}$ ion is above the hollow site (see Fig. S4 of the Supplemental Material [19]).

DFT $+U$ calculations have been performed to study the magnetic exchange coupling of $\mathrm{Cr}$-porphin (Cr-P) molecules to the $\mathrm{Co}(001)$ surface. For details of the employed computational method, see Refs. [7,22]. The Coulomb $U$ and exchange $J$ parameters were 4 and $1 \mathrm{eV}$, respectively [23], the generalized-gradient approximation [24] was used, and van der Waals dispersion corrections were taken into account [25]. The Cr-P molecule is computed to adsorb preferably on top of a hollow site position, at a distance of $3.1 \AA$ of the Cr ion from the topmost Co layer. The chromium ion is calculated to be in a high-spin state $S \approx 2$ and to couple antiferromagnetically to the Co substrate magnetization. In Fig. 3(b) the local magnetic density of states (DOS) of Cr-P adsorbed on the $\mathrm{Co}(001)$ substrate is presented. The $d_{x y}, d_{x z}, d_{y z}$, and $d_{z^{2}}$ orbitals are singly occupied, i.e., the $\mathrm{Cr}$ ion has a less than half-filled $3 d$ shell. The $d_{\pi}$ orbitals [note that only one of $\left(d_{x z}, d_{y z}\right)$ is shown] are practically degenerated, as dictated by the symmetry for square-planar complexes. The $d_{x^{2}-y^{2}}$ orbital of $\mathrm{Cr}$ is split into bonding and antibonding molecular orbitals; the bonding molecular orbital at about $-4.2 \mathrm{eV}$ is partly occupied, due to $\sigma$ donation from the ligands to the metal center (and back bonding) [26]. The magnetic isosurface around the $\mathrm{Cr}$ ion has an elongated shape along the normal direction ( $z$ axis) [Fig. 3(a)], which is also indicative of a predominantly unoccupied $d_{x^{2}-y^{2}}$ orbital. The inset of Fig. 3(b) shows the DOS for the minority (red) and majority (blue) spin bands of the spin-polarized Co substrate; the AFM coupling of the Cr spin to the Co substrate spin is apparent from its occupied DOS being in the spin-down channel (gray shaded area). The hybridization between the molecule's and the substrate's electronic states is best seen from the charge density plot (see Fig. S4 of the Supplemental Material [19]).

Our findings provide an exciting case compared to those known from earlier studies of metalloporphyrins and metallophthalocyanines. The so-far observed coupling mechanisms can be classified in four schemes with regard to the magnetic interaction between the metal-organic species and the FM substrates: (i) direct FM exchange coupling between the central ion of the molecule and the substrate [11,27], and three types of indirect magnetic exchange coupling, which are (ii) indirect $180^{\circ}$ AFM coupling via oxygen or graphene $[5,6,12]$, (iii) indirect $90^{\circ} \mathrm{FM}$ coupling $[1,2,16,22]$, and (iv) oscillatory Ruderman-Kittel-Kasuya-Yosida (RKKY) exchange coupling through an interlayer with a substrate [28]. We note that the XMCD signal of CrTPP molecules on a $\mathrm{Cl}$ covered Co substrate shows no magnetic coupling at the limit of

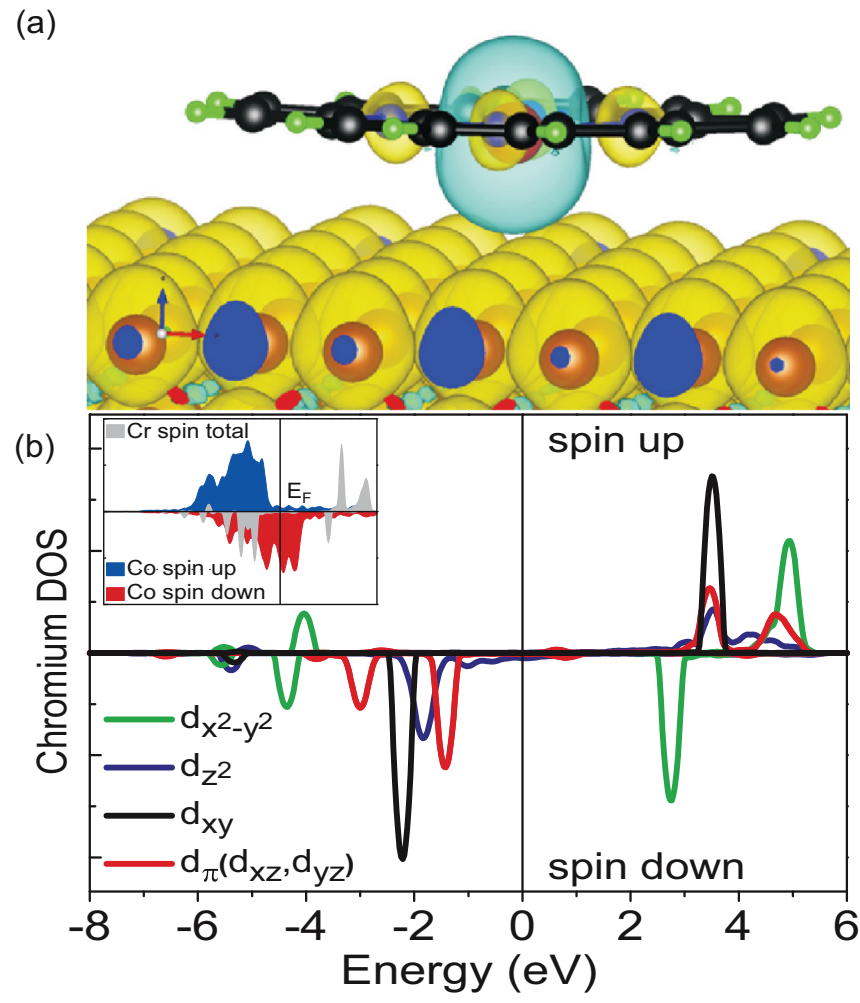

FIG. 3. (Color) DFT $+U$ calculations of Cr-P adsorbed on a $\mathrm{Co}(001)$ substrate. (a) Plot of the magnetic spin-density isosurfaces of the $\mathrm{Cr}-\mathrm{P} / \mathrm{Co}$ system. Yellow (blue) colored hypersurfaces depict spinmajority (spin-minority) magnetization. (b) Spin-resolved density of states calculations of $\mathrm{Cr} d$ orbitals reveal AFM coupling of the spin moment of the $\mathrm{Cr}$ ion to that of $\mathrm{Co}$. The inset shows the spin-resolved density of states of the Co substrate with spin-up (blue) and spin-down (red) bands, and the spin-polarized total DOS of Cr in gray.

detection (cf. Fig. S1 of the Supplemental Material [19]). We therefore exclude the $180^{\circ}$ indirect exchange coupling via chlorine as the origin of the AFM coupling between CrTPP and the Co substrate. Hence, none of these listed schemes can appropriately describe the AFM coupling of CrTPP to $\mathrm{Co}(001)$ observed here. We assign this effect to the less than half-filled $d$ shell in the Cr(II)TPP admolecules. Employing the Goodenough-Kanamori-Anderson (GKA) theory [29] as guidance, we can model the AFM coupling of CrTPP to $\mathrm{Co}(001)$ as an $\mathrm{AFM}$ indirect $90^{\circ}$ exchange coupling.

The $\mathrm{Cr}$ ion in the CrTPP, not the CrTPPCl molecule, is found in the oxidation state $2+$ on the Co surface, implying a $\left(d_{x y}\right)^{1},\left(d_{\pi}\right)^{2}\left[\left(d_{x z}\right)^{1},\left(d_{y z}\right)^{1}\right],\left(d_{z^{2}}\right)^{1}$ electronic configuration (left part of Fig. 4) and a high-spin state $S=2$, as evidenced by the DFT $+U$ calculations (Fig. 3). The magnetic coupling of the $\mathrm{Cr} d$-shell electrons can occur either by a direct exchange interaction with the out-of-plane orbitals of the Co substrate [11,27] or via an indirect exchange interaction through the nitrogen orbitals $[16,20,22]$. Since a strong direct exchange interaction (overlap between the $d_{z^{2}}$ orbital of $\mathrm{Cr}$ and out-of-planes orbitals of $\mathrm{Co}$ ) would be accompanied by FM coupling [11], the AFM coupling is assigned to an indirect coupling via the nitrogen electrons. We note, however, that a broadening of the $\mathrm{Cr} d_{z^{2}}$ in DOS occurs in Fig. 3(b), which indicates a competing, but weaker, direct interaction. Upon 


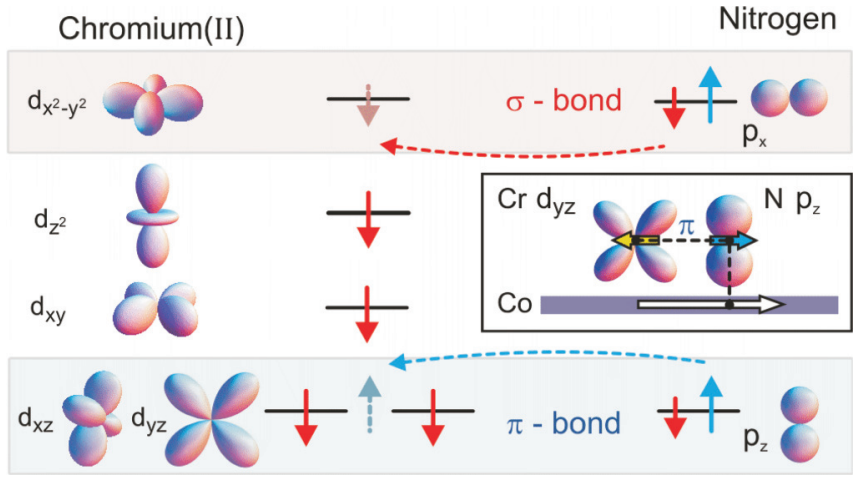

FIG. 4. (Color) Left: $3 d$ orbitals of the Cr ion and their electron occupations in $\mathrm{CrTPP} / \mathrm{Co}(001)$ according to Hund's rules. Due to indirect coupling (via nitrogen) to the spin-up polarized Co substrate, the Cr electrons exhibit a spin-down orientation (red arrows). Right: The nitrogen $2 p_{x}$ and $2 p_{z}$ orbitals. The $\mathrm{N} p$ electrons show an imbalance of spin-down (red arrows) and spin-up (blue arrows) electrons due to interaction with the Co substrate. The effective electron exchange through the $\sigma$ and $\pi$ bonds is indicated by the dashed arrows. Box: Schematic side view of the indirect $90^{\circ}$ exchange coupling of the spins in $\mathrm{CrTPP} / \mathrm{Co}(001)$.

contact with the FM substrate the nitrogen atoms in the porphyrin macrocycle obtain a small net magnetic moment which is aligned parallel to the substrate magnetization [see the nitrogen spin density in Fig. 3(a)] [7,27]. The bonding to the Co out-of-plane orbitals occurs via interaction of the spin-down electron from the $\mathrm{N} p_{z}$ orbitals. The $\mathrm{N} p_{z}$ orbitals also form a $\pi$ bond with the $\operatorname{Cr} d_{x z}, d_{y z}$ orbitals (lower row of Fig. 4). This configuration is equivalent to a $90^{\circ}$ cation-anion-cation interaction scheme (cf. the box in Fig. 4) and is intuitively treated within the GKA model [29]. There, the cations involved in the interaction comprise the $\mathrm{Cr}$ ion on one side and the Co substrate on the other side, while the nitrogens within the porphyrin ring provide the anion. The formation of the $\pi$ bond between the $\mathrm{Cr} d_{x z}, d_{y z}$ orbitals and the $\mathrm{N} p_{z}$ orbitals leads to an exchange interaction between the spin-up electron from nitrogen and the $\mathrm{Cr}$ ion (lower row of Fig. 4). The substrate induced spin-up polarization of the nitrogen $p$ electrons, together with Pauli's exclusion principle, force the $\mathrm{Cr} d_{\pi}$ electrons to align their spins antiparallel to the electrons of the $\mathrm{N} p_{z}$ orbitals. The intra-atomic FM interactions induce a parallel spin alignment of all $\mathrm{Cr} d$-shell electrons. As a result, the total electron spin of $\mathrm{Cr}$ is AFM coupled to the Co substrate, as observed experimentally and in our DFT $+U$ calculations.

It is instructive to discuss the role of an extra electron in the $d$ shell of the magnetic ion in a similar environment, e.g., in MnTPP on Co(001). Starting with the $d^{4}$ configuration, the fifth $d$ electron of such a system will occupy the $d_{x^{2}-y^{2}}$ orbital, which forms a $\sigma$-type bond with the $\mathrm{N} p_{x}$ orbital. The overlap of the $\mathrm{Cr} d_{x^{2}-y^{2}}$ and the $\mathrm{N} p_{x}$ orbitals leads to sharing the spin- down electron from nitrogen by both orbitals. Note that the electrons in the $\mathrm{N} p_{x}$ orbitals do not contribute to the bonding with the Co substrate. Due to the intra-atomic FM interaction between nitrogen orbitals, the $\mathrm{N} p_{x}$ orbital possesses a spinup polarization. Thus, the additional $d$ electron in the $d_{x^{2}-y^{2}}$ orbital will experience a strong exchange interaction with the polarized $\mathrm{N} p_{x}$ electrons, resulting in a parallel alignment of magnetic moments of $\mathrm{N}$ and $\mathrm{Cr}$ atoms. The $\sigma$ bond is much stronger than the $\pi$ bond and hence the spin polarization of the $d_{x^{2}-y^{2}}$ orbital is intra-atomically transferred to the remaining $d$-shell electrons of Mn. Consequently, a FM coupling arises in the magnetic ion with one more electron as compared to the CrTPP studied here. This is observed experimentally as well as theoretically in our DFT $+U$ calculations for MnTPP on $\mathrm{Co}(001)[1,7,27]$. These considerations show that the $d$-shell occupation, which differs by one between $\mathrm{Cr}$ and $\mathrm{Mn}$, plays a crucial role in the magnetic coupling of porphyrins (and possibly also for phthalocyanines) to magnetic substrates.

In summary, we reported an AFM exchange coupling between a magnetic $\mathrm{Cr}$-porphyrin molecule and a bare FM Co substrate. The discovered AFM coupling is assigned to the less than half-filled $d$ shell of the high-spin Cr ion in the Cr(II)TPP molecules. In agreement with the Goodenough-KanamoriAnderson (GKA) theory, the indirect exchange coupling between the $\mathrm{Cr}$ ion and Co substrate occurs dominantly via the $\mathrm{N}$ atoms in the porphyrin. Our findings demonstrate that the magnetic coupling of molecules to surface substrates can be controlled by external stimuli. This may occur, for example, by electron transfer between a coordinating ligand and the metal center which modifies its initial $d^{4}\left(d^{5}\right)$ configuration to a final $d^{5}\left(d^{4}\right)$ configuration and correspondingly re-orients its magnetization from FM to AFM alignment with respect to the substrate or vice versa. A transition from FM to AFM coupling was recently demonstrated when MnTPP molecules on a Co substrate are ligated with a strong electron $\pi$-acceptor, nitric oxide, transforming the $\mathrm{Mn}$ ion from a $d^{5}$ to a $d^{4}$ configuration [7]. Such control of the interface exchange coupling could be used to engineer suitable organic-inorganic spin interfaces for applications in molecular spintronic and/or logic devices.

We gratefully acknowledge financial support from the Swiss National Science Foundation (SNSF), Swiss Nanoscience Institute (SNI), Holcim Foundation for the Advancement of Scientific Research, Switzerland, and from the Swedish-Indian Research Links Programme. The authors sincerely thank Rolf Schelldorfer for technical support and Milos Baljozovic for scientific discussion. Part of this work was performed at the Surface/Interface:Microscopy (SIM) beamline of the Swiss Light Source (SLS), Paul Scherrer Institut, Villigen, Switzerland. N.B. thanks K.N. Ganesh (IISER Pune) for support during the beamtimes at the SLS. Support from the Swedish National Infrastructure for Computing (SNIC) is also acknowledged.
[1] A. Scheybal, T. Ramsvik, R. Bertschinger, M. Putero, F. Nolting, and T. A. Jung, Chem. Phys. Lett. 411, 214 (2005).
[2] H. Wende, M. Bernien, J. Luo, C. Sorg, N. Ponpandian, J. Kurde, J. Miguel, M. Piantek, X. Xu, P. Eckhold, W. Kuch, 
K. Baberschke, P. M. Panchmatia, B. Sanyal, P. M. Oppeneer, and O. Eriksson, Nat. Mater. 6, 516 (2007).

[3] L. Bogani and W. Wernsdorfer, Nat. Mater. 7, 179 (2008).

[4] M. Mannini, F. Pineider, P. Sainctavit, C. Danieli, E. Otero, C. Sciancalepore, A. M. Talarico, M.-A. Arrio, A. Cornia, D. Gatteschi, and R. Sessoli, Nat. Mater. 8, 194 (2009).

[5] M. Bernien, J. Miguel, C. Weis, M. E. Ali, J. Kurde, B. Krumme, P. M. Panchmatia, B. Sanyal, M. Piantek, P. Srivastava, K. Baberschke, P. M. Oppeneer, O. Eriksson, W. Kuch, and H. Wende, Phys. Rev. Lett. 102, 047202 (2009).

[6] D. Chylarecka, C. Wäckerlin, T. K. Kim, K. Müller, F. Nolting, A. Kleibert, N. Ballav, and T. A. Jung, J. Phys. Chem. Lett. 1, 1408 (2010).

[7] C. Wäckerlin, K. Tarafder, D. Siewert, J. Girovsky, T. Hählen, C. Iacovita, A. Kleibert, F. Nolting, T. A. Jung, P. M. Oppeneer, and N. Ballav, Chem. Sci. 3, 3154 (2012).

[8] C. Wäckerlin, J. Nowakowski, S.-X. Liu, M. Jaggi, D. Siewert, J. Girovsky, A. Shchyrba, T. Hählen, A. Kleibert, P. M. Oppeneer, F. Nolting, S. Decurtins, T. A. Jung, and N. Ballav, Adv. Mater. 25, 2404 (2013).

[9] A. Mugarza, C. Krull, R. Robles, S. Stepanow, G. Ceballos, and P. Gambardella, Nat. Commun. 2, 490 (2011).

[10] J. Brede, N. Atodiresei, S. Kuck, P. Lazić, V. Caciuc, Y. Morikawa, G. Hoffmann, S. Blügel, and R. Wiesendanger, Phys. Rev. Lett. 105, 047204 (2010).

[11] S. Javaid, M. Bowen, S. Boukari, L. Joly, J.-B. Beaufrand, X. Chen, Y. J. Dappe, F. Scheurer, J.-P. Kappler, J. Arabski, W. Wulfhekel, M. Alouani, and E. Beaurepaire, Phys. Rev. Lett. 105, 077201 (2010).

[12] C. F. Hermanns, K. Tarafder, M. Bernien, A. Krüger, Y.-M. Chang, P. M. Oppeneer, and W. Kuch, Adv. Mater. 25, 3473 (2013).

[13] A. Lodi Rizzini, C. Krull, T. Balashov, J. J. Kavich, A. Mugarza, P. S. Miedema, P. K. Thakur, V. Sessi, S. Klyatskaya, M. Ruben, S. Stepanow, and P. Gambardella, Phys. Rev. Lett. 107, 177205 (2011).

[14] C. Wäckerlin, K. Tarafder, J. Girovsky, J. Nowakowski, T. Hählen, A. Shchyrba, D. Siewert, A. Kleibert, F. Nolting,
P. M. Oppeneer, T. A. Jung, and N. Ballav, Angew. Chem. Int. Ed. 52, 4568 (2013).

[15] N. Ballav, C. Wäckerlin, D. Siewert, P. M. Oppeneer, and T. A. Jung, J. Phys. Chem. Lett. 4, 2303 (2013).

[16] D. Chylarecka, T. K. Kim, K. Tarafder, K. Müller, K. Gödel, I. Czekaj, C. Wäckerlin, M. Cinchetti, M. E. Ali, C. Piamonteze, F. Schmitt, J.-P. Wüstenberg, C. Ziegler, F. Nolting, M. Aeschlimann, P. M. Oppeneer, N. Ballav, and T. A. Jung, J. Phys. Chem. C 115, 1295 (2011).

[17] C. Theil, J. van Elp, and F. Folkmann, Phys. Rev. B 59, 7931 (1999).

[18] S. Kuck, M. Prostak, M. Funk, M. Bröring, G. Hoffmann, and R. Wiesendanger, J. Vac. Sci. Technol. A 28, 795 (2010).

[19] See Supplemental Material at http://link.aps.org/supplemental/ 10.1103/PhysRevB.90.220404 for XMCD and STM data on CrTPPCl-Cl-Co and DFT $+U$ charge density maps of Cr-P/Co.

[20] J. V. Barth, Annu. Rev. Phys. Chem. 58, 375 (2007).

[21] W. Auwärter, K. Seufert, F. Klappenberger, J. Reichert, A. Weber-Bargioni, A. Verdini, D. Cvetko, M. Dell'Angela, L. Floreano, A. Cossaro, G. Bavdek, A. Morgante, A. P. Seitsonen, and J. V. Barth, Phys. Rev. B 81, 245403 (2010).

[22] P. M. Oppeneer, P. M. Panchmatia, B. Sanyal, O. Eriksson, and M. E. Ali, Prog. Surf. Sci. 84, 18 (2009).

[23] P. M. Panchmatia, B. Sanyal, and P. M. Oppeneer, Chem. Phys. 343, 47 (2008).

[24] J. P. Perdew, K. Burke, and M. Ernzerhof, Phys. Rev. Lett. 77, 3865 (1996).

[25] S. Grimme, J. Comput. Chem. 27, 1787 (2006).

[26] R. K. Hocking, E. C. Wasinger, Y.-L. Yan, F. M. F. deGroot, F. A. Walker, K. O. Hodgson, B. Hedman, and E. I. Solomon, J. Am. Chem. Soc. 129, 113 (2007).

[27] Md. E. Ali, B. Sanyal, and P. M. Oppeneer, J. Phys. Chem. C 113, 14381 (2009).

[28] Y.-S. Fu, Q.-K. Xue, and R. Wiesendanger, Phys. Rev. Lett. 108, 087203 (2012).

[29] J. B. Goodenough, Magnetism and the Chemical Bond (Wiley, New York, 1963). 